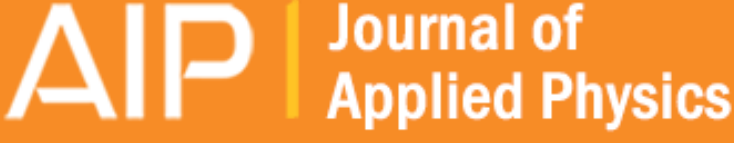

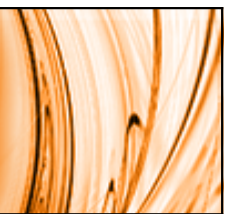

\section{Theoretical study of electronic transport properties of a graphene-silicene bilayer}

G. R. Berdiyorov, H. Bahlouli, and F. M. Peeters

Citation: Journal of Applied Physics 117, 225101 (2015); doi: 10.1063/1.4921877

View online: http://dx.doi.org/10.1063/1.4921877

View Table of Contents: http://scitation.aip.org/content/aip/journal/jap/117/22? ver=pdfcov

Published by the AIP Publishing

\section{Articles you may be interested in}

Observing and tuning the density distribution of localized states of monolayer graphene oxide by using external electric field

Appl. Phys. Lett. 106, 131103 (2015); 10.1063/1.4916745

Electronic and structural properties at the interface between CuPc and graphene

J. Appl. Phys. 117, 013701 (2015); 10.1063/1.4904087

Modulation of the electron transport properties in graphene nanoribbons doped with BN chains

AIP Advances 4, 067123 (2014); 10.1063/1.4883236

Fano effects in electron transport through an armchair graphene nanoribbon with one line defect

J. Appl. Phys. 113, 233701 (2013); 10.1063/1.4811438

Electron transport in quantum antidots made of four-terminal graphene ribbons

J. Appl. Phys. 106, 104303 (2009); 10.1063/1.3259408

Frustrated by

old technology?

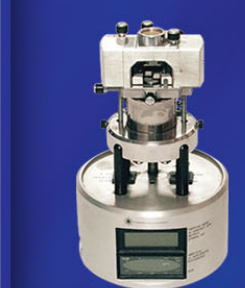

Is your AFM dead

and can't be repaired?

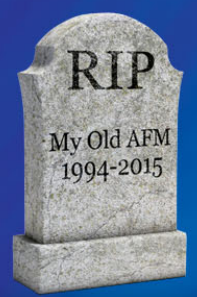

Sick of bad customer support?

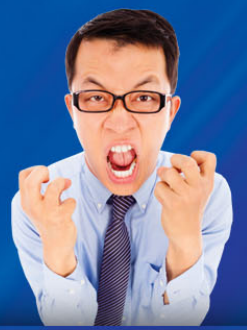

It is time to upgrade your AFM Minimum $\$ 20,000$ trade-in discount for purchases before August 31st

Asylum Research is today's technology leader in AFM

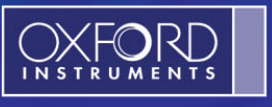




\title{
Theoretical study of electronic transport properties of a graphene-silicene bilayer
}

\author{
G. R. Berdiyorov, ${ }^{1}$ H. Bahlouli, ${ }^{2,3}$ and F. M. Peeters ${ }^{4}$ \\ ${ }^{1}$ Qatar Environment and Energy Research Institute, Qatar Foundation, P.O. Box 5825, Doha, Qatar \\ ${ }^{2}$ Department of Physics, King Fahd University of Petroleum and Minerals, 31261 Dhahran, Saudi Arabia \\ ${ }^{3}$ Saudi Center for Theoretical Physics, 31261 Dhahran, Saudi Arabia \\ ${ }^{4}$ Departement Fysica, Universiteit Antwerpen, Groenenborgerlaan 171, B-2020 Antwerpen, Belgium
}

(Received 31 January 2015; accepted 16 May 2015; published online 8 June 2015)

\begin{abstract}
Electronic transport properties of a graphene-silicene bilayer system are studied using density-functional theory in combination with the nonequilibrium Green's function formalism. Depending on the energy of the electrons, the transmission can be larger in this system as compared to the sum of the transmissions of separated graphene and silicene monolayers. This effect is related to the increased electron density of states in the bilayer sample. At some energies, the electronic states become localized in one of the layers, resulting in the suppression of the electron transmission. The effect of an applied voltage on the transmission becomes more pronounced in the layered sample as compared to graphene due to the larger variation of the electrostatic potential profile. Our findings will be useful when creating hybrid nanoscale devices where enhanced transport properties will be desirable. (C) 2015 AIP Publishing LLC.
\end{abstract}

[http://dx.doi.org/10.1063/1.4921877]

\section{INTRODUCTION}

Recent advances in nanofabrication technology made it possible to create new monolayer materials and to reveal their properties under different conditions. Among those materials, graphene - a single layer of hexagonally arranged carbon atoms-is the most attractive material due to its unique physical, chemical, and mechanical properties. ${ }^{1}$ According to the theory, ${ }^{2,3}$ monolayer honeycomb structures similar to graphene can be obtained from the other group-IV elements and their binary compounds. Among them the most interesting material is silicene, ${ }^{4-7}$ a silicon analogue of graphene, which has the potential for practical applications, in part, due to silicon's compatibility with existing electronics infrastructure. Due to the similarity of the lattice structures, silicene has similar electronic properties as graphene. ${ }^{6,8-10}$ For example, the band structure of silicene resembles that of graphene with bands crossing linearly at the Fermi level, resulting in a massless Dirac-like fermion character for its charge carriers. However, unlike graphene, silicene is not stable in a flat configuration: it forms a slightly buckled monolayer structure due to the fact that $s p^{3}$ hybridization for $\mathrm{Si}$ is more stable than $s p^{2}$ hybridization. ${ }^{4,9,11}$

Despite the number of theoretical reports predicting the stability of silicene, free standing silicene has not been experimentally realized to date. Deposition of silicon on metal surfaces that interact weakly with the silicon atoms is considered to be one of the promising routes to synthesize silicene (see Ref. 12 for review). However, the structure of silicene on metallic surfaces was found to be much more complicated than that of the theoretically predicted free-standing silicene: ${ }^{13-22}$ A plethora of very different crystallographic $\mathrm{Si}$ structures were obtained depending on the growth conditions and the particular atomic arrangements of the substrate surface, where the interaction with the substrate and epitaxial strain becomes the determining factors. Consequently, the electronic properties of silicene on metallic surfaces are considerably different from those of free standing silicene. ${ }^{17,19,23,24}$

Very recently, graphene has been proposed to be a possible substrate for silicene growth. ${ }^{25,26}$ Furthermore, graphene is a very good thermal conductor which will be an important asset for device applications. Due to the weak coupling between silicene and graphene, both structural and electronic properties of silicene are less affected in contrast to the case of metallic substrates. ${ }^{27,28}$ Theory predicts that the characteristics of silicene in graphene-silicene multilayers are very close to those predicted for standalone buckled silicene. For example, the silicene Dirac cone is preserved and it does not interfere with the graphene Dirac point. ${ }^{28}$ In addition, such graphene-silicene-superlattice was predicted to be stable well above room temperature. ${ }^{28}$

In this work, we used first-principles density-functional theory (DFT) calculations in combination with the nonequilibrium Green's function formalism to study electronic transport properties of graphene-silicene bilayer. This approach has recently been used to study the electronic transport in silicene nanoribbons with hydrogen termination. ${ }^{29}$ Here, we found that the graphene-silicene bilayer shows enhanced transmission as compared to the sum of the transmissions of individual graphene and silicene sheets at energies near the Fermi level. The reason of such larger transmission is traced back to the appearance of additional electronic states that are extended into the interplanar spacing. However, away from the Fermi energy some of the states become localized in one of the layers, which reduce the electron transmission across the sample.

\section{COMPUTATIONAL DETAILS}

We first optimized isolated silicene and graphene using DFT calculations ${ }^{30}$ within the generalized gradient 
approximation (GGA) of Perdew-Burke-Ernzerhof (PBE) to represent the exchange-correlation energy. ${ }^{31}$ The Brillouin zone integration was performed using $6 \times 6 \times 6 \mathrm{k}$-point sampling. ${ }^{32}$ The electrostatic potentials were determined on a real-space grid with a mesh cutoff energy of $150 \mathrm{Ry}$ and double-zeta-double-polarized basis sets of local numerical orbitals were employed for all atoms. Once optimized, we constructed an interface between silicene and graphene and minimized the system energy by relaxing the interplanar spacing. Grimme's DFT-D2 empirical dispersion correc$\operatorname{tion}^{33}$ to the PBE was used to account for van der Waals interactions, which is known to be very important for the accurate description of such multilayer structures. To avoid a large unit cell due to the mismatch between the lattice parameters of silicene and graphene, we strained both graphene and silicene layer with less than $1.5 \%$. The unit cell consists of 16 silicon atoms and 36 carbon atoms (see the inset of Fig. 1). To study the transport properties of the system, we have constructed a two probe device geometry with 64 silicon atoms and 144 carbon atoms in the active layer, as shown in Fig. 3. Quantum transport properties of the system are calculated using the nonequilibrium Green's function formalism with the Brillouin zone sampled with $(6,6,100)$ points within the Monkhorst-Pack $k$-point sampling scheme. All simulations were conducted by using the first-principles computational package Atomistix toolkit (ATK). ${ }^{34}$

\section{STRUCTURAL PROPERTIES}

We first conducted structural optimization for free standing silicene starting from the planar honeycomb arrangement of $\mathrm{Si}$ atoms, which is known to be the least energetic and not stable configuration. ${ }^{9}$ The convergence for energy was taken as $10^{-5} \mathrm{eV}$ between two consecutive steps, and the condition that the maximum force on each atom was less than $0.01 \mathrm{eV} / \AA$. During the optimization, the system was relaxed to a buckled structure with buckling parameter $\delta=0.52 \AA$ and bond length $2.287 \AA$, which are in good agreement with the pervious DFT/PBE reports. ${ }^{35,36}$ Once optimized, we created an interface between the silicene and graphene sheets. The simulation unit cell consists of 16 silicon atoms and 36 carbon atoms (see the inset of Fig. 1). Next, we minimized the system energy by varying the

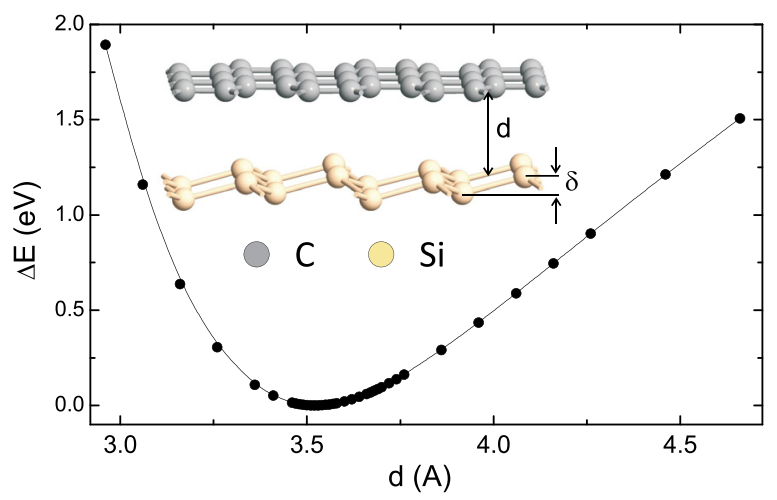

FIG. 1. The total energy difference of graphene-silicene bilayer system as a function of interplanar distance $d$. Inset shows the minimum energy configuration of the system with the parameters $d=3.52 \AA$ and $\delta=0.52 \AA$. interplanar distance $d$. For that purpose, we performed explicit calculations for the energy as a function of distance $d$ taking into account dispersive interactions. Such energydistance optimizations are cumbersome in practice, especially when calculating more complex structures. Calculated variation of the system energy as a function of the interplanar distance is presented in Fig. 1. The minimum energy state is observed for $d=3.52 \AA$ (see the inset in Fig. 1), which is slightly larger than the interplanar distance in graphene-silicene multilayers $d=3.42 \AA .^{28}$ The system energy increases rapidly with decreasing interplanar distance, whereas the slope of the $\Delta E(d)$ curve is smaller at larger distances. We tested whether the same level of accuracy can be achieved in a more straightforward manner, that is, by a direct force and stress relaxation. The convergence of the directly optimized lattice parameters towards the ones retrieved from explicit energy-volume optimization is obtained with a difference less than $0.01 \AA$.

It was well known that both free standing graphene and silicene have Dirac Fermion character, which is reflected in the linear band crossing in the band structure of the systems. As was also predicted in pervious DFT calculations (see, e.g., Refs. 27 and 28), the most unique property of these two dimensional materials (i.e., the Dirac fermion-like electronic structure) is preserved in the graphene-silicene bilayer structure. This behavior is also reflected in the density of states (DOS) of the system as a linear dependence of DOS on the electron energy close to the Dirac point (see Fig. 2).

\section{ZERO VOLTAGE TRANSMISSION}

Based on the optimized graphene-silicene structure, we constructed a device geometry (see Fig. 3), which consists of left and right regions and the central (scattering) region (i.e., a two probe configuration). On the left and right parts of the sample, we put metallic electrodes with in plane periodic

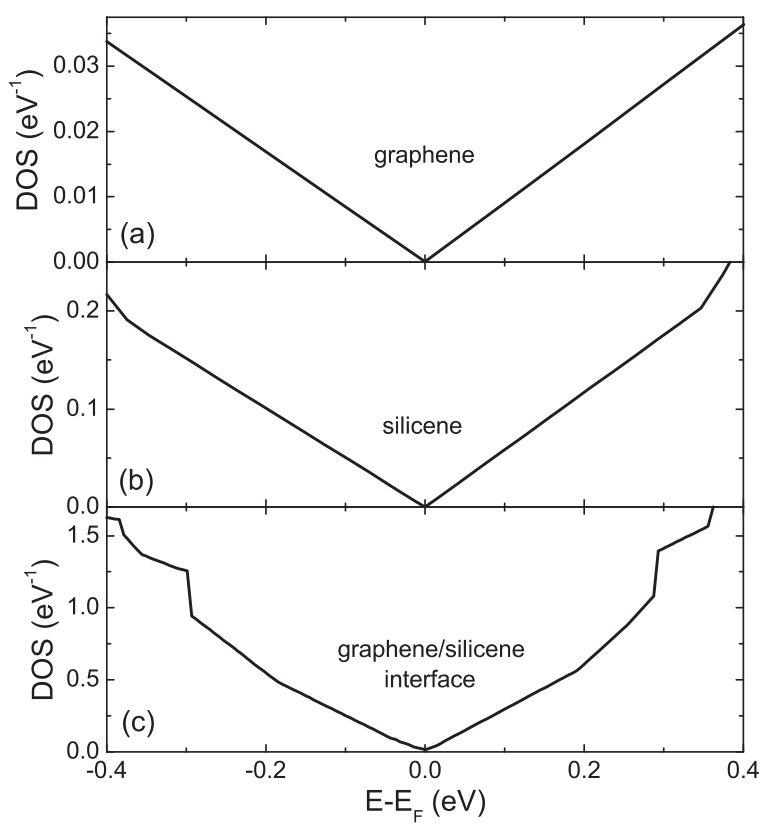

FIG. 2. Density of states of graphene (a), silicene (b), and graphene-silicene interface structure (c). 


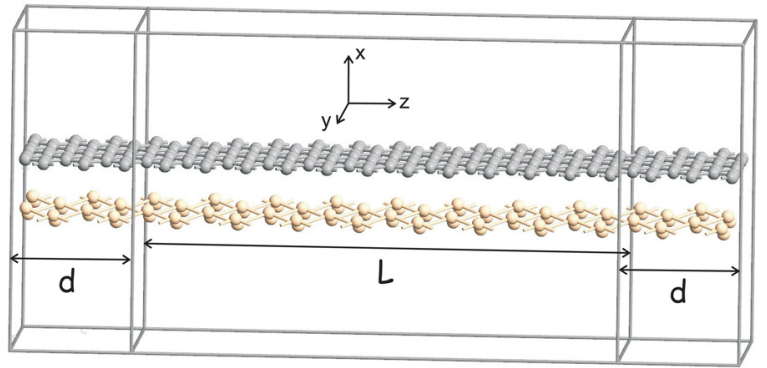

FIG. 3. Device geometry: graphene-silicene bilayer with 144 carbon and 64 silicon atoms. Periodic boundary conditions are applied along the $x$ - and $y$-directions and electronic transport occurs along the $z$-direction through the metallic contacts (of size $d=7.55 \AA$ ). Vacuum spacing of more than $15 \AA$ are left along the $x$-direction to avoid the formation of a graphene/silicene superlattice structure. $^{27}$

boundary conditions perpendicular to the sample metal contacts. The metallic contact is taken as an electron gas with a fixed chemical potential. The system is periodic along the $x$ - and $y$-directions. As a typical example, we consider the system with 144 carbon and 64 silicon atoms in the scattering region and the electrode size $d=7.55 \AA$. This size of the active region $(L=30.2 \AA)$ was large enough to avoid electrode interactions, at the same time providing accurate modelling with a reasonable computational cost. For reference, we also consider the device configurations consisting of only graphene and silicene layers. We start by calculating the equilibrium transport properties (no voltage biasing) of the system. Figure 4(a) shows the zero voltage transmission spectra, $T(E)$, as a function of the electronic energy for the considered samples. Dashed-dotted-green curve shows the sum of the transmission of individual graphene and silicene devices. Zero energy is set to be the Fermi level of the system. Solid-black curve in this figure shows the transmission spectrum of graphene, which exhibits a sequence of steps and finite transmission at the Fermi level, which are typical for graphene. Solid-black curve in Fig. 4(b) shows the device density of states (DDOS) of graphene, which shows a strong correlation with the transmission coefficient, especially as far as the location of their peaks is concerned. This indicates that there is a correspondence between the energy levels of the system and the transmission spectrum. Dashed-red curve in Fig. 4(a) shows the zero bias transmission spectrum of silicene. It shows enhanced transmission for the charge carriers of all considered energies. This is consistent with the recent first-principles calculations. ${ }^{29}$ Finally, dotted-blue curve in Fig. 4(a) shows $T(E)$ of the graphene-silicene bilayer system. Interestingly, this system shows enhanced transmission near the Fermi level. In fact, the transmission is larger than the sum of the transmissions of separated graphene and silicene samples [compare dotted-blue and dashed-dotted-green curves in Fig. 4(a)]. Such an enhancement is accompanied by an increase in DDOS of the system [see Fig. 4(b)]. However, away from the Fermi energy the electron transmission in the bilayer system becomes comparable to or smaller than the sum of the transmissions in the other two systems. This happens despite the similar or even larger DDOS in the bilayer system [see Fig. 4(b)]. It is known that localization of electronic states is one of the main factors affecting the
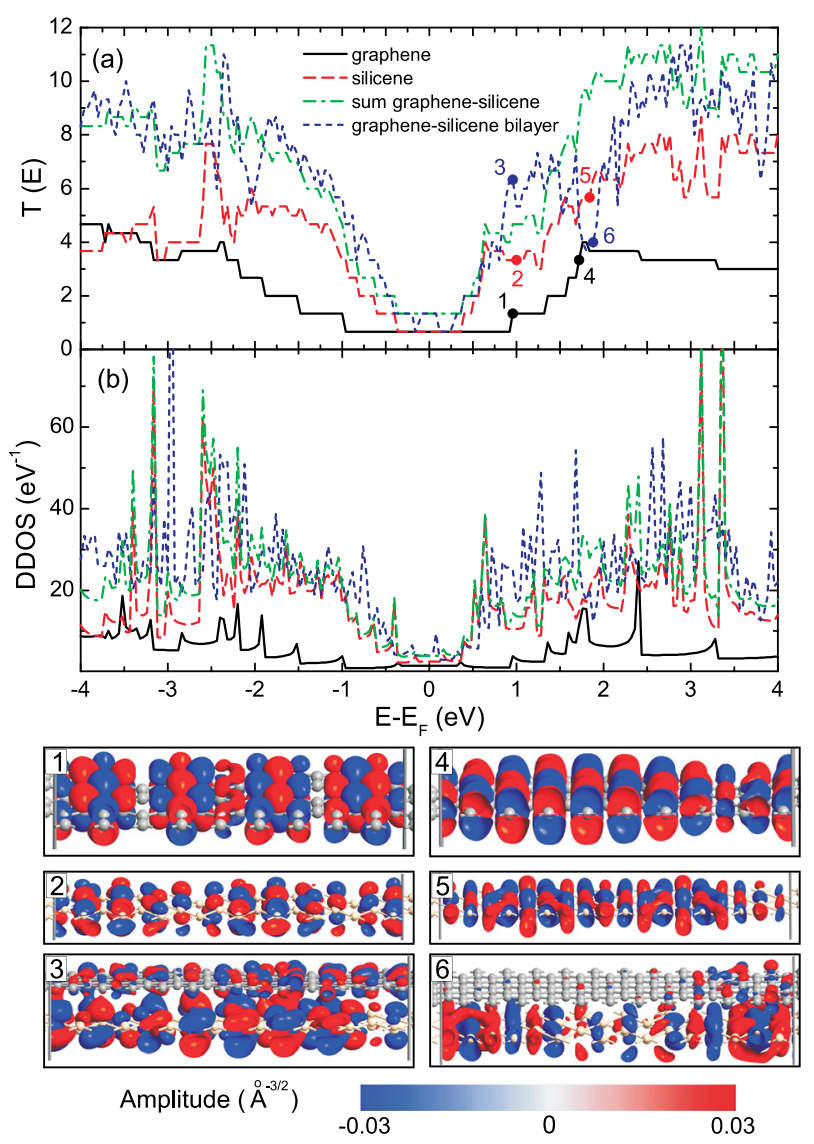

FIG. 4. (a) Zero bias transmission spectrum $(T(E))$ of pristine graphene (solid-black curve), pristine silicene (dashed-red curve), and the graphenesilicene bilayer system (dotted-blue curve) as a function of electron energy (the Fermi energy is taken at $E=0$ ). Dashed-dotted-green curve shows the sum of the transmissions of graphene and silicene systems. (b) Device density of states of all three systems as a function of energy with zero energy broadening. Panels 1-6: isosurface plots of the projected self-consistent Hamiltonian eigenstates of graphene (1 and 4), silicene (2 and 5), and graphene-silicene bilayer (3 and 6) corresponding to the energies indicated on the $T(E)$ curves.

electronic transmission in nanoscale devices. To see if this mechanism could explain the enhanced transmission in the coupled graphene-silicene bilayer, we calculated and compared the projected self-consistent Hamiltonian (PSH) eigenstates of all three systems at different energies. This can be achieved by projecting the self-consistent Hamiltonian onto the Hilbert space spanned by the basis functions of the central atoms. The eigenstates of the PSH are associated with the poles of the Green's function and, in general, roughly correspond to the peaks in the transmission spectrum. Panels 1-6 in Fig. 4 show some samples of isosurface plots of the PSH eigenstates corresponding to energies indicated on the transmission curves. For small energies in the conduction band, all the states are delocalized between the electrodes (panels 1 and 2). In the bilayer system, the electronic states are extended between the layers with higher electron density on the silicene layer (panel 3). At higher energies, most of the states are on the silicene layer, where they are localized near the electrodes (panel 6). Such localization reduces the probability of the electrons to cross the system, i.e., it results in a smaller transmission. Thus, the graphene-silicene system shows enhanced transport of 
electrons near the Fermi energy as compared to pristine silicene and graphene due to the enhanced DDOS. However, deeper inside the conduction and valence bands, the electron transmission in the bilayer system becomes smaller due to the energies of localized electronic states.

\section{FINITE VOLTAGE BIASING}

In what follows, we study the response of the considered samples to finite voltage biasing. As a typical example, we present in Fig. 5 the transmission spectrum of graphene (a), silicene (b), and coupled graphene-silicene (c) samples for two values of the applied voltage (the transmission spectra at zero voltage are given for reference). The variation of the transmission spectra with the applied voltage is clearly seen for all the three samples. As a general trend, the transmission is reduced for all electron energies; in fact, electrons can be totally reflected near the Fermi energy. Energy dependent asymmetry is preserved at finite biasing. The area under the transmission spectrum decreases further with increasing applied voltage (blue-dotted curve), implying that the conductance also decreases rapidly according to Landauer's current formula. ${ }^{37}$ The most prominent feature of the voltage dependence of the transmission is the appearance of a well separated peak in the transmission spectrum at the Fermi energy, which spreads to energies away from the Fermi level

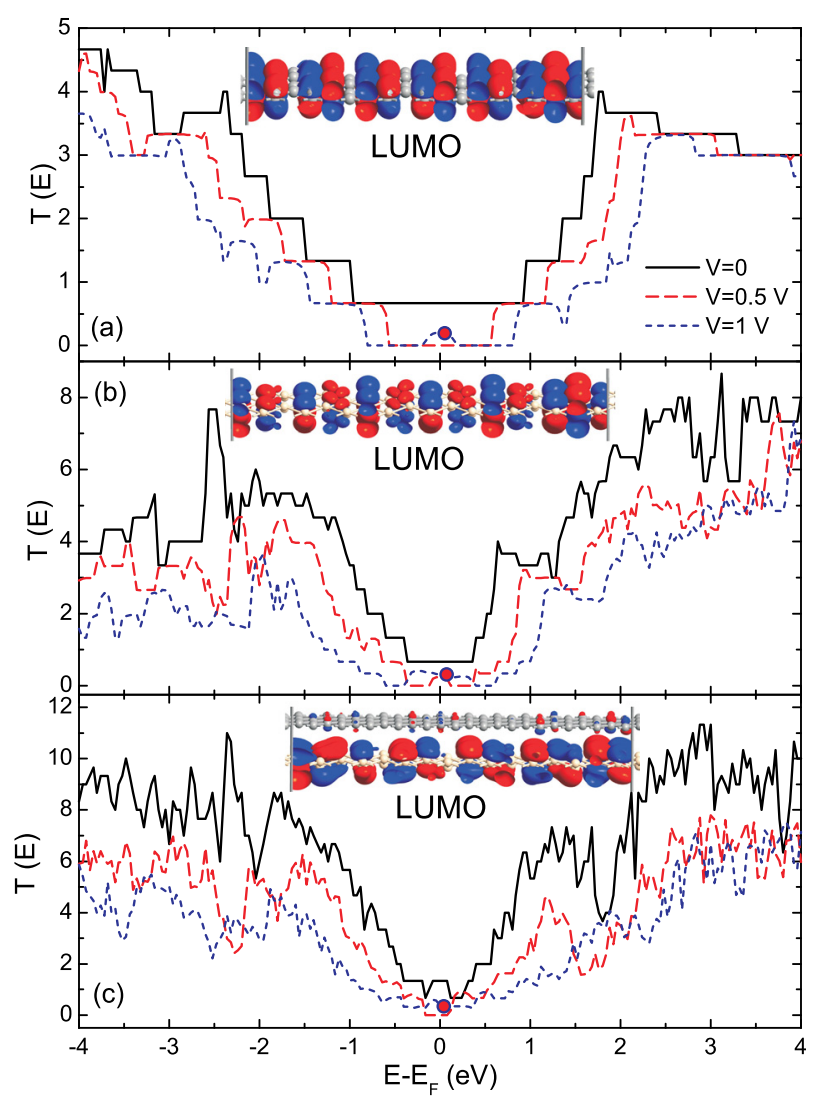

FIG. 5. Transmission spectrum of graphene (a), silicene (b), and coupled graphene-silicene bilayer (c) as a function of energy (with respect to the Fermi energy) for zero voltage biasing (solid-black curves) and for finite voltage biasing with $\Delta V=0.5 \mathrm{~V}$ (dashed-red curves) and $\Delta V=1 \mathrm{~V}$ (dotted blue curves). Insets show the LUMOs of the samples at $\Delta V=1 \mathrm{~V}$, the energies of which are indicated by symbols on the transmission curves. with further increase in the applied potential difference. This indicates the opening of new transport channels for electrons when applying a finite voltage (see the lowest unoccupied molecular orbital (LUMO) of the system in the insets of Fig. 5). The graphene-silicene system also shows a similar response to voltage biasing: (i) reduction of the electron transmission (in fact, the reduction is larger as compared to the other samples) and (ii) the appearance of finite transmission near the Fermi level at larger biasing. Calculations of the electron transmission eigenstates show that some states near the Fermi energy becomes more extended, which enhances the electronic transmission through the system. Thus, all considered systems show similar response to the applied voltage with new features in the transmission spectrum. However, the effect of voltage biasing is less pronounced in graphene as compared to the other two samples. This is consistent with recent DFT calculations, ${ }^{29}$ where the effect of the applied voltage was related to the change in the electrostatic potential profile. The latter depends on the ionic size and becomes more pronounced in silicene as compared to the graphene sample. ${ }^{29}$ This is also seen in Fig. 6, where we plot the electrostatic difference potential along the transport direction for all three samples at $\Delta V=1 \mathrm{~V}$. The potential profile of the silicene sample shows larger oscillations as compared to that of the graphene sample, which implies higher scattering at these sites according to the Büttiker formalism. The amplitude of the oscillations becomes slightly larger in the case of the coupled graphene-silicene bilayer.

\section{CONCLUSION}

First-principles DFT calculations in combination with the nonequilibrium Green's functional formalism have been performed to study the structural and electronic transport properties of coupled graphene-silicene bilayer system. The system shows enhanced transmission as compared to both individual silicene and graphene samples. In addition, depending on the energy of the electrons, the electron transmission in the bilayer system can be larger as compared to the sum of the transmissions of separated graphene and silicene monolayers. We relate this effect to the increased electron density of states in the bilayer sample. Localization of the electronic states has also been observed, which results in

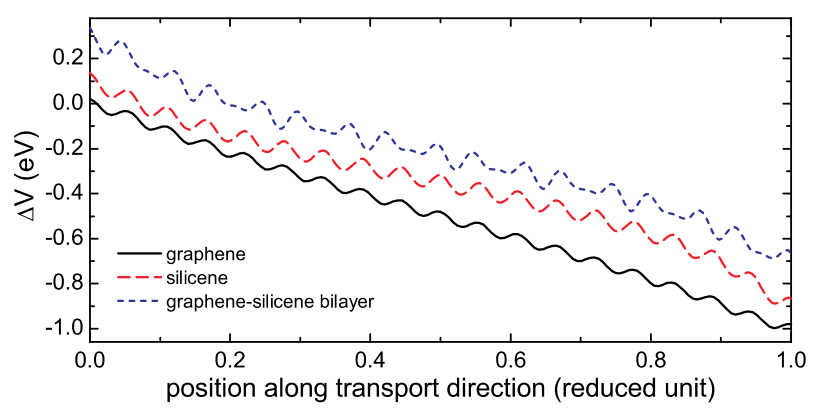

FIG. 6. Electrostatic difference potential along the transport direction for graphene (solid-black curve), silicene (dashed-red curve), and coupled graphene-silicene bilayer (dotted-blue curve) for a potential difference across the sample of $\Delta V=1 \mathrm{~V}$. The results obtained for silicene and graphenesilicene bilayer are shifted vertically by $0.1 \mathrm{eV}$ and $0.3 \mathrm{eV}$, respectively. 
the suppression of the electron transmission. The effect of applied voltage on the transmission becomes more pronounced in the layered sample as compared to graphene due to the larger variation of the electrostatic potential profile. Our theoretical findings can be helpful for potential future engineering of nanoscale devices where enhanced transport properties will be an asset.

\section{ACKNOWLEDGMENTS}

H. B. and F. M. P. acknowledge support from King Fahd University of Petroleum and Minerals, Saudi Arabia, under the RG1329-1 and RG1329-2 DSR Projects.

${ }^{1}$ K. S. Novoselov, A. K. Geim, S. V. Morozov, D. Jiang, Y. Zhang, S. V. Dubonos, I. V. Grigorieva, and A. A. Firsov, Science 306, 666 (2004); K. S. Novoselov, A. K. Geim, S. V. Morozov, D. Jiang, M. I. Katsnelson, I. V. Grigorieva, S. V. Dubonos, and A. A. Firsov, Nature (London) 438, 197 (2005); A. K. Geim and K. S. Novoselov, Nat. Mater. 6, 183 (2007); C. Neto, F. Guinea, N. M. R. Peres, K. S. Novoselov, and A. K. Geim, Rev. Mod. Phys. 81, 109 (2009).

${ }^{2}$ H. Şahin, S. Cahangirov, M. Topsakal, E. Bekaroglu, E. Akturk, R. T. Senger, and S. Ciraci, Phys. Rev. B 80, 155453 (2009).

${ }^{3}$ L. Pan, H. J. Liu, Y. W. Wen, X. J. Tan, H. Y. Lv, J. Shi, and X. F. Tang, Phys. Lett. A 375, 614 (2011).

${ }^{4}$ K. Takeda and K. Shiraishi, Phys. Rev. B 50, 14916 (1994).

${ }^{5}$ Y. Zhang, Y. W. Tan, H. L. Stormer, and P. Kim, Nature (London) 438, 201 (2005).

${ }^{6}$ G. Guzmán-Verri and L. C. Lew Yan Voon, Phys. Rev. B 76, 075131 (2007).

${ }^{7}$ G. R. Berdiyorov and F. M. Peeters, RSC Adv. 4, 1133 (2014).

${ }^{8}$ A. O'Hare, F. V. Kusmartsev, and K. I. Kugel, Nano Lett. 12, 1045 (2012).

${ }^{9}$ S. Cahangirov, M. Topsakal, E. Akturk, H. Şahin, and S. Ciraci, Phys. Rev. Lett. 102, 236804 (2009).

${ }^{10}$ S. Lebègue and O. Eriksson, Phys. Rev. B 79, 115409 (2009).

${ }^{11}$ U. Röthlisberger, W. Andreoni, and M. Parrinello, Phys. Rev. Lett. 72, 665 (1994).

${ }^{12}$ A. Kara, H. Enriquez, A. P. Seitsonen, L. C. Lew Yan Voon, S. Vizzini, B. Aufray, and H. Oughaddou, Surf. Sci. Rep. 67, 1 (2012).

${ }^{13}$ C. Leandri, G. Le Lay, B. Aufray, C. Girardeaux, J. Avila, M. E. Davila, M. C. Asensio, C. Ottaviani, and A. Cricenti, Surf. Sci. 574, L9 (2005).
${ }^{14}$ B. Lalmi, H. Oughaddou, H. Enriquez, A. Kara, S. Vizzini, B. Ealet, and B. Aufray, Appl. Phys. Lett. 97, 223109 (2010).

${ }^{15}$ B. Aufray, A. Vizzini, S. Oughaddou, H. Leandri, C. Ealet, and G. Le Lay, Appl. Phys. Lett. 96, 183102 (2010).

${ }^{16}$ P. De Padova, C. Quaresima, C. Ottaviani, P. M. Sheverdyaeva, P. Moras, C. Carbone, D. Topwal, B. Olivieri, A. Kara, H. Oughaddou, B. Aufray, and G. Le Lay, Appl. Phys. Lett. 96, 261905 (2010).

${ }^{17}$ P. Vogt, P. De Padova, C. Quaresima, J. Avila, E. Frantzeskakis, M. C. Asensio, A. Resta, B. Ealet, and G. Le Lay, Phys. Rev. Lett. 108, 155501 (2012).

${ }^{18}$ B. Feng, Z. Ding, S. Meng, Y. Yao, X. He, P. Cheng, L. Chen, and K. Wu, Nano Lett. 12, 3507 (2012).

${ }^{19}$ L. Chen, C.-C. Liu, B. Feng, X. He, P. Cheng, Z. Ding, S. Meng, Y. Yao, and K. Wu, Phys. Rev. Lett. 109, 056804 (2012).

${ }^{20}$ H. Jamgotchian, Y. Colignon, N. Hamzaoui, B. Ealet, J. Y. Hoarau, B. Aufray, and J. P. Bibérian, J. Phys.: Condens. Matter 24, 172001 (2012).

${ }^{21}$ L. Chen, H. Li, B. Feng, Z. Ding, J. Qiu, P. Cheng, K. Wu, and S. Meng, Phys. Rev. Lett. 110, 085504 (2013).

${ }^{22}$ L. Meng, Y. Wang, L. Zhang, S. Du, R. Wu, L. Li, Y. Zhang, G. Li, H. Zhou, W. A. Hofer, and H.-J. Gao, Nano Lett. 13, 685 (2013).

${ }^{23}$ Y.-P. Wang and H.-P. Cheng, Phys. Rev. B 87, 245430 (2013).

${ }^{24}$ C.-L. Lin, R. Arafune, K. Kawahara, M. Kanno, N. Tsukahara, E. Minamitani, Y. Kim, M. Kawai, and N. Takagi, Phys. Rev. Lett. 110, 076801 (2013).

${ }^{25}$ R. Zhou, L. C. Lew Yan Voon, and Y. Zhuang, J. Appl. Phys. 114, 093711 (2013).

${ }^{26}$ G. R. Berdiyorov, M. Neek-Amal, F. M. Peeters, and A. C. T. van Duin, Phys. Rev. B 89, 024107 (2014).

${ }^{27}$ Y. Zhang and R. Tsu, Nanoscale Res. Lett. 5, 805 (2010).

${ }^{28}$ M. Neek-Amal, A. Sadeghi, G. R. Berdiyorov, and F. M. Peeters, Appl. Phys. Lett. 103, 261904 (2013).

${ }^{29}$ S. Yamacli, J. Nanopart. Res. 16, 2576 (2014).

${ }^{30}$ W. Kohn and L. J. Sham, Phys. Rev. 140, A1133 (1965).

${ }^{31}$ J. P. Perdew, K. Burke, and M. Ernzerhof, Phys. Rev. Lett. 77, 3865 (1996).

${ }^{32}$ H. J. Monkhorst and J. D. Pack, Phys. Rev. B 13, 5188 (1976).

${ }^{33}$ S. Grimme, J. Comput. Chem. 27, 1787 (2006).

${ }^{34}$ See http://www.quantumwise.com for distribution by QuantumWise Company, Copenhagen, Denmark.

${ }^{35}$ T. H. Osborn, A. A. Farajian, O. V. Pupysheva, R. S. Aga, and L. C. L. Y. Voon, Chem. Phys. Lett. 511, 101 (2011).

${ }^{36}$ S. Trivedi, A. Srivastava, and R. Kurchania, J. Comput. Theor. Nanosci. 11, 781 (2014).

${ }^{37}$ S. Datta, Quantum Transport: Atoms to Transistors (Cambridge University Press, UK, 2005). 